\title{
Three-dimensional ultrasonic imaging in multilayered media
}

\author{
Martin H. Skjelvareid*, Tomas Olofsson ${ }^{\dagger}$ and Yngve Birkelund* \\ *Department of Physics and Technology, University of Tromsø, Norway \\ $\dagger$ Department of Engineering Sciences, Uppsala University, Sweden
}

\begin{abstract}
The synthetic aperture focusing technique is a method for focusing ultrasonic scans used in nondestructive testing. Traditionally, the technique has mainly been used for contact testing, where the speed of sound is constant throughout the whole medium, but a number of recently proposed algorithms have extended the technique to multilayered media. One important application for such multilayer methods is immersion testing, where an object is immersed in water and the transducer is scanned within the water layer. Similarly, a multilayer technique is useful for inline ultrasound inspection of pipelines filled with liquid, for example water or oil.

The multilayer methods have so far only been applied to the 2-dimensional line scan case, but in this paper we extend the the focusing procedure to the 3-dimensional surface scan case. The proposed method is based on the frequency-domain Phase Shift Migration algorithm, which is both conceptually simpler and more computationally efficient than similar time-domain methods.

The performance of the focusing algorithm is tested on a three-layer structure, consisting of acrylic glass and aluminum blocks immersed in water. Several small flat-bottom holes in each block are used to simulate point scatterers, and the scatterer responses are studied using both B- and C-scan presentation. The lateral resolution of the focused image is shown to be approximately equal to half the transducer diameter, independent of both depth and layer.
\end{abstract}

Keywords: ultrasound, SAFT, synthetic aperture, immersion, multilayer, phase shift migration PACS: $43.35 .+\mathrm{d}, 43.60 .+\mathrm{d}$

\section{INTRODUCTION}

Synthetic aperture focusing is used in radar, sonar, seismic and ultrasound imaging. The technique combines pulse-echo measurement performed in several positions, and creates a reflectivity image with a significantly improved lateral resolution.

Within the field of ultrasonic non-destructive testing, the technique was initially developed during the 1970s and 1980s, and it was termed the "Synthetic Aperture Focusing Technique" (SAFT) 4]. SAFT was originally applied to contact scans, where it can be assumed that the waves propagate through a single, uniform medium. However, in a multilayered geometry, the wave velocity in each layer will in general be different, and this has to be taken into account by the focusing algorithm. This is for example the case for immersion scans, where there is a water layer present between the ultrasound transducer and the object under test.

A number of SAFT algorithms adapted to multilayered geometries have recently been proposed [5, 7, 6]. The algorithms have so far only been applied to the line scan case, but in this paper, the Phase Shift Migration algorithm proposed in [5] is extended to the three-dimensional surface scan case, enabling three-dimensional imaging.

\section{THEORY}

It is assumed that a series of pulse-echo measurements are acquired for all $(x, y)$ positions over the plane $z=Z$, and that all reflectors are located in the half-space $z>Z$. The set of measurements are denoted $p(t, x, y, Z)$, where $t$ denotes the two-way delay time of the pulse. The Fourier transform of the recorded wave 
field is given by

$$
P\left(\omega, k_{x}, k_{y}, Z\right)=\frac{1}{(2 \pi)^{3}} \iint_{-\infty}^{\infty} \int_{\infty} p(t, x, y, Z) e^{-i\left(k_{x} x+k_{y} y-\omega t\right)} d x d y d t,
$$

where $\frac{1}{(2 \pi)^{3}}$ is a normalization constant. It can be shown [8] that within a region of constant sound speed, $c$, the wave field can be extrapolated to any depth $Z+\Delta z$ by multiplication with a complex exponential,

$$
P\left(\omega, k_{x}, k_{y}, Z+\Delta z\right)=P\left(\omega, k_{x}, k_{y}, Z\right) \cdot \exp \left(i k_{z} \Delta z\right)
$$

where $k_{z}$ is given by

$$
k_{z}\left(\omega, k_{x}, k_{y}, c\right)=-\operatorname{sgn}(\omega) \cdot \sqrt{\frac{\omega^{2}}{c^{2}}-k_{x}^{2}-k_{y}^{2}} .
$$

The sign function ensures that the $k_{z}$ value represents a wave traveling in the negative z direction. An inverse Fourier transform is used to obtain the extrapolated wave field in time-space coordinates,

$$
p(t, x, y, Z+\Delta z)=\iint_{-\infty}^{\infty}\left[P\left(\omega, k_{x}, k_{y}, Z\right) \cdot e^{i k_{z} \Delta z}\right] e^{i\left(k_{x} x+k_{y} y-\omega t\right)} d k_{x} d k_{y} d \omega .
$$

Derivations of migration algorithms for the monostatic case are often based on the exploding reflector model [1, which simplifies the inverse imaging problem. This model is also applied here. The scatterers in the region to be imaged are assumed to be sources of acoustic energy, so that the acoustic signals recorded by the transducer are the result of a one-way transmission rather than a two-way transmission and reflection. The propagation delay for the waves remain the same under the exploding reflector model if the effective speed of sound, $\hat{c}$, is assumed to be half that of the actual speed of sound, $\hat{c}=c / 2$.

Under the exploding reflector model, the wave field from a scatterer is maximally concentrated in space at the "time of explosion" ( $\mathrm{t}=0)$. Thus, a focused image can be obtained from a recorded and Fourier transformed pulse-echo measurement $P\left(\omega, k_{x}, k_{y}, z\right)$ by setting the speed of sound in Eq. 3 equal to $\hat{c}=c / 2$, and applying the imaging condition $t=0$ to Eq. 4 .

$$
I(x, y, z)=\iint_{-\infty}^{\infty} \int^{\infty} P\left(\omega, k_{x}, k_{y}, z\right) e^{i\left(k_{x} x+k_{y} y\right)} d k_{x} d k_{y} d \omega .
$$

Note that this reduces the inverse transform with respect to $\omega$ to a simple integral over $\omega$. Equation 2 can be used iteratively to extrapolate the wave field to all depths of interest, and Eq. 5 can subsequently be used to create a focused image plane for each depth. This is the Phase Shift Migration (PSM) originally proposed by Gazdag [2]. If the region to be imaged consists of multiple layers, the speed of sound is a function of depth, $c(z)$. The PSM algorithm can handle such variation in speed of sound, since the $k_{z}$ wave number given by Eq. 3 can be updated during the iterative extrapolation to new depths; $k_{z}\left(\omega, k_{x}, k_{y}, \frac{c(z)}{2}\right)$.

\section{EXPERIMENT}

To test the ability of the algorithm to focus images from multilayered media, an experiment with two test blocks with flat bottom holes was conducted. The test blocks were made of poly(methyl methacrylate), also known as acrylic glass, and each block had four flat-bottom holes with $3 \mathrm{~mm}$ diameter and $20 \mathrm{~mm}$ lateral spacing. The holes in the acrylic glass block were 6, 12, 18 and $24 \mathrm{~mm}$ deep, and the holes in the aluminum block were 10, 20, 30 and $40 \mathrm{~mm}$ deep. The acrylic glass block was placed on top of the aluminum block, and the arrangement was immersed in water to create a three-layer structure. The blocks were also placed with a $10 \mathrm{~mm}$ offset in both the $x$ and $y$ directions to ensure that the holes in the aluminum block would not be shadowed by the holes in the acrylic glass block. The experimental setup is shown in Fig. 11, where the left figure shows the setup from the side, and the right figure shows the blocks from above. The $y$ position of the holes in the acrylic glass block and the aluminum block is indicated with $y_{1}$ and $y_{2}$, respectively. 

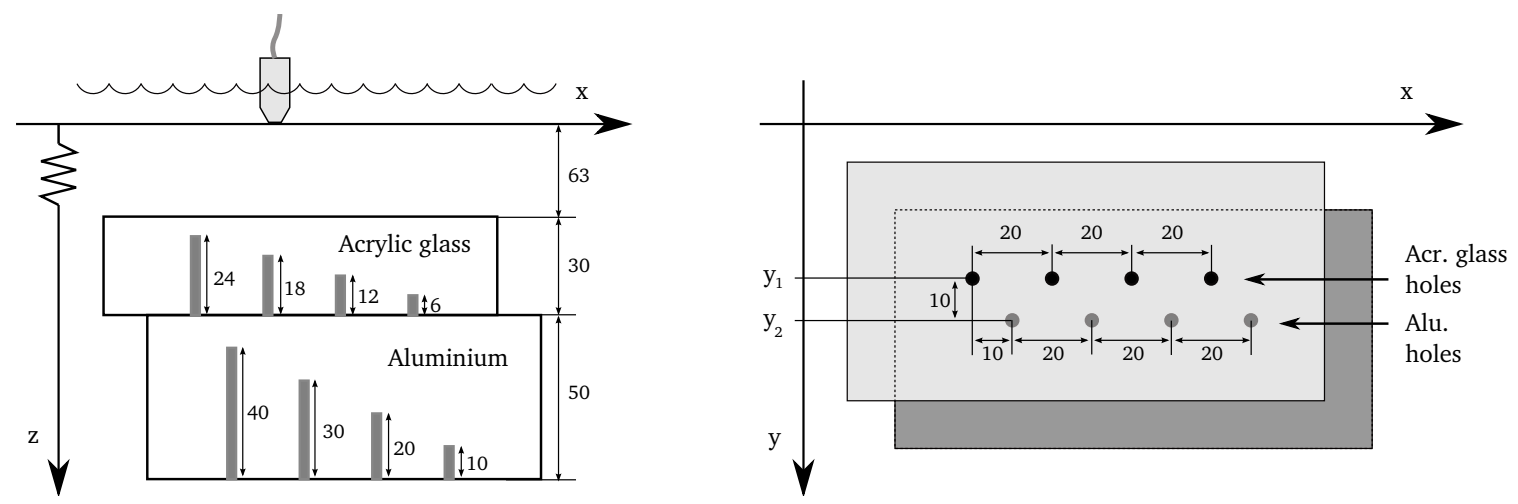

Figure 1. Experimental setup, acrylic glass and aluminum blocks with flat-bottom holes. All dimensions are in mm.
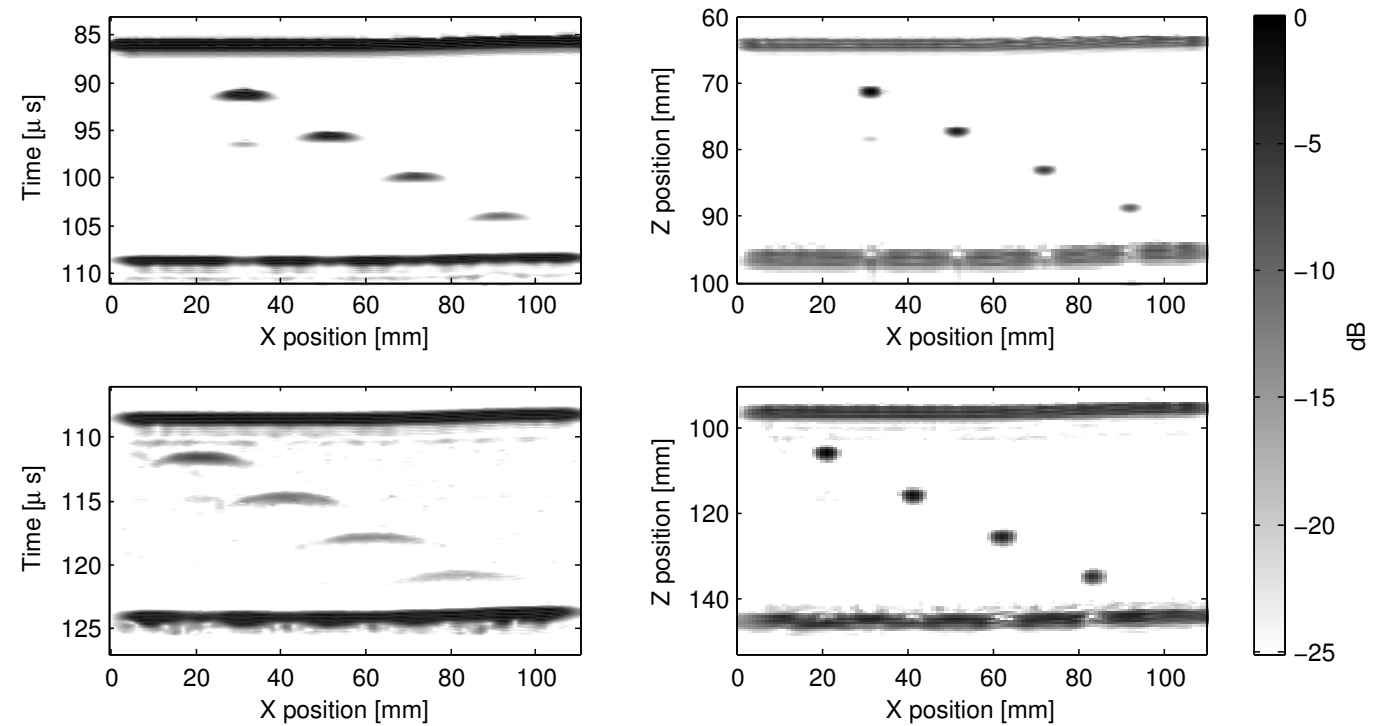

Figure 2. 2D slices from the raw and focused 3D images. Left: Raw data. Right: Focused image. Top: Slices from the acrylic glass block, $y=y_{1}$. Bottom: Slices from the aluminum block, $y=y_{2}$. The images have been normalized relative to the highest amplitude in each image.

The two upper plots in Fig. 2 show slices of the raw data and focused image from the acrylic glass block, centered on $y_{1}$, and the two lower plots show corresponding slices from the aluminum block, centered on $y_{2}$. It is seen that in the raw data, the response from the flat-bottom holes becomes gradually broader with increasing depth. This effect, which reduces the lateral resolution of the image, is due to the divergence of the ultrasound beam. Compared to the raw data, the corresponding focused images are seen to have a significantly improved lateral resolution. The width of the response from each scatterer is also approximately the same, independent of depth of layer.

A common way to visualize ultrasonic 3D scans is to plot the maximum back-scattered amplitude within a time/depth interval for each measurement position. This is called a C-scan. In Fig. 3 the two top plots show C-scans from within the acrylic glass block, and the bottom plots show C-scans from the aluminum block. The left plots are obtained from the raw data, and the right plots are obtained from the focused 3D image.

These plots illustrate that synthetic aperture focusing results in an improved lateral resolution along both the $x$ and $y$ axis. The diameter of the response at $-3 \mathrm{~dB}$ from the peak was estimated for all of the scatterers, and it was found that the mean diameters were $2.3 \mathrm{~mm}$ in acrylic glass and $2.7 \mathrm{~mm}$ in aluminum, respectively. 

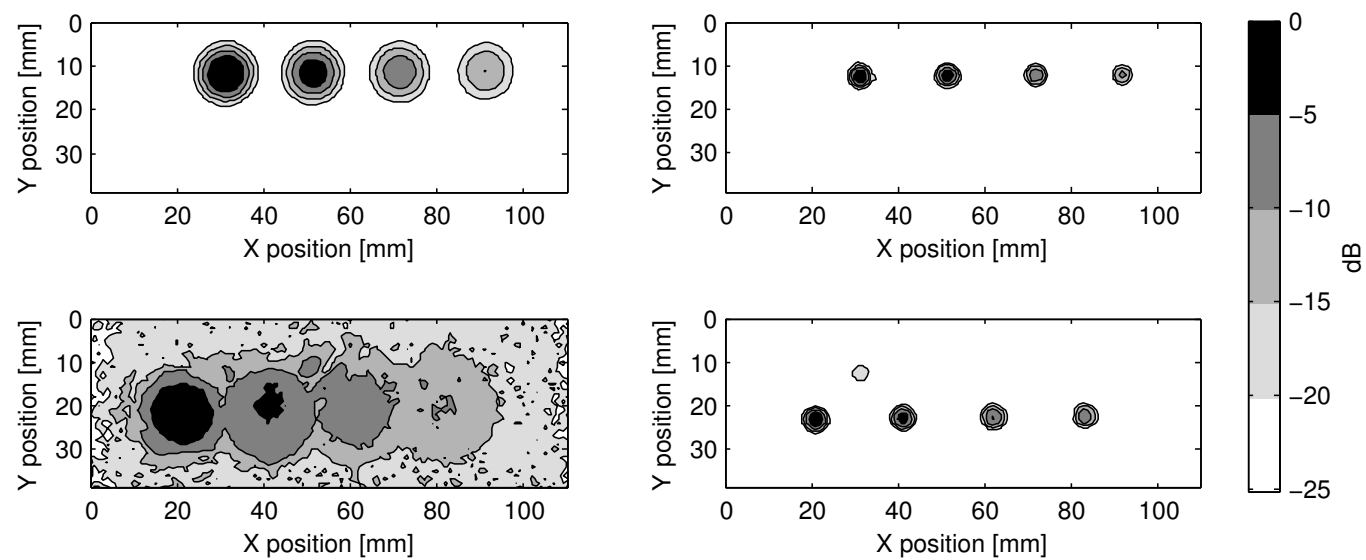

Figure 3. C-scans obtained from 3D data sets. Left: Raw data. Right: Focused image. Top: C-scan from within acrylic glass block. Bottom: C-scan from within aluminum block. The images have been normalized relative to the highest amplitude in each image.

This corresponds well to the theoretical resolution of approximately half the transducer diameter [3], which in this case is $3 \mathrm{~mm}$.

In the lower left plot, it can be seen that backscattered reflections from the aluminum layer are almost buried in background noise. This is due to the large transmission losses at the interfaces between the layers. However, in the aluminum layer C-scan for the focused image, the responses from the flat bottom holes are well above the background noise. This is because ultrasonic reflections are added together coherently in the synthetic aperture algorithm, while at the same time canceling out random noise. There is also an additional reflection seen at approximately $(x, y)=(30,10) \mathrm{mm}$ in the aluminum layer. This is due to a multiple reflection of the hole at this position in the acrylic glass layer.

\section{CONCLUSIONS}

It has been shown that the Phase Shift Migration algorithm can be successfully extended to three-dimensional ultrasonic imaging. This enables the use of synthetic aperture techniques in horizontally layered geometries. The lateral resolution of the focused image is shown to be approximately half the transducer diameter, independent of depth or layer.

\section{REFERENCES}

1. Jon F. Claerbout. Imaging the Earth's Interior. Blackwell Scientific Publications, 1985.

2. Jenö Gazdag. Wave equation migration with the phase-shift method. Geophysics, 43(7):1342-1351, 1978.

3. Peter T. Gough and David W. Hawkins. Unified framework for modern synthetic aperture imaging algorithms. International Journal of Imaging Systems and Technology, 8(4):343-358, 1998.

4. K.J. Langenberg, M. Berger, Th. Kreutter, K. Mayer, and V. Schmitz. Synthetic aperture focusing technique signal processing. NDT International, 19(3):177-189, June 1986.

5. Tomas Olofsson. Phase shift migration for imaging layered objects and objects immersed in water. IEEE Transactions on Ultrasonics, Ferroelectrics, and Frequency Control, 57(11):2522-2530, November 2010.

6. Martin H. Skjelvareid, Tomas Olofsson, Yngve Birkelund, and Yngvar Larsen. Synthetic aperture focusing of ultrasonic data from multilayered media using an omega-k algorithm. IEEE Transactions on Ultrasonics, Ferroelectrics, and Frequency Control, 58(5):1037-1048, May 2011.

7. Martin Hansen Skjelvareid and Yngve Birkelund. Ultrasound imaging using multilayer synthetic aperture focusing. In ASME 2010 Pressure Vessels and Piping Conference, volume 5, pages 379-387, 2010.

8. Earl G. Williams. Fourier Acoustics: Sound Radiation and Nearfield Acoustical Holography. Academic Press, 1999. 